\title{
Interstellar Extinction in the Direction of Young Open Star Clusters
}

\author{
Alok K. Durgapal* \\ Center of Advanced Study, Department of Physics, D. S. B. Campus, Kumaun University Nainital, India
}

\begin{abstract}
The total to selective extinction law in optical and near-IR wavelengths for twenty young open star clusters namely Berkeley 7, Collinder 69, Hogg 10, NGC 2362, Czernik 43, NGC 6530, NGC 6871, Bochum 10, Haffner 18, IC 4996, NGC 2384, NGC 6193, NGC 6618, NGC 7160, Collinder 232, Haffner 19, NGC 2401, NGC 6231, NGC 6823 , NGC 7380 have been studied. It is found that fifteen clusters follow normal extinction law and five clusters show an anomalous behavior.
\end{abstract}

Keywords: Star cluster: Reddening, interstellar dust- Interstellar extinction

\section{INTRODUCTION}

The interstellar dust which is remnant of star formation process can transmit and redirect the light of stars ([2]). As a result it becomes very difficult to determine accurate distances and magnitudes of astronomical objects ([11]); to overcome this difficulty we must have knowledge of composition of interstellar dust in every line of sight. The interstellar extinction is caused either by general interstellar medium (ISM) or by localized region of higher mean density ([9], [15]). Study of interstellar extinction provides information about components of the molecular cloud from which a star was formed. Young stars contain dust around them so young open star clusters are the ideal objects for extinction study ([5], [8], [10], [16], [17]). First started with Trumpler in 1920 and then many investigators studied extinction in the Galaxy. [7] investigated extinction law in two different stellar aggregates and found different extinction curves for these two aggregates. From this study they confirmed the presence of two populations of grains. [6] studied twenty big OB associations situated at different parts of Galaxy and found that all the associations have similar type of interstellar matter. [13] studied interstellar extinction around 200 stars located within four open clusters and found an anomalous extinction law. [1] derived a mean extinction law for both diffuse and dense interstellar medium. [17] found presence of non-uniform extinction along 15 open star clusters. [11] studied 14 open clusters and found that for $\lambda \geq \mathrm{J}_{\lambda}$, dust along these objects follow normal interstellar law. [4] studied the distribution of interstellar matter near the Galactic plane and found that $\sim 90$ percent of absorbing material lies within $-50^{\circ} \leq \mathrm{b} \leq 50^{\circ}$ of the Galactic plane. As discussed above there are many studies regarding the interstellar extinction but yet for many clusters there is very limited knowledge of distribution of dust in the Milky Way. To map all the dust in the Milky Way more studies are required. Also for accurate distance measurements of stars, one needs the value of extinction in every direction. In present study we analyzed interstellar extinction law for twenty young open star clusters. In section 2, we have described the data sources and cluster selection criteria. In section 3, we presented interstellar extinction law for the twenty open star clusters and in last section we concluded our analysis.

\section{CLUSTER SELECTION AND OBSERVATIONAL DATA}

For present analysis we selected twenty young open star clusters namely Berkeley 7, Collinder 69 , Hogg 10, NGC 2362, Czernik 43, NGC 6530, NGC 6871, Bochum 10, Haffner 18, IC 4996, NGC 2384, NGC 6193, NGC 6618, NGC 7160, Collinder 232, Haffner 19, NGC 2401, NGC 6231, NGC 6823, NGC 7380 from the WEBDA catalogue having age less than 50 Myr. The optical data in UBVRI filters are taken from WEBDA database and near-IR data in JHK band are retrieved from 2MASS catalogue. To analyze interstellar extinction law, we selected early type stars in these clusters with the help of colour-colour diagram. To determine the intrinsic colours of the stars, we used Q-method ([3]). The optical data have error $\sim 0.01 \mathrm{mag}$ in $\mathrm{V}$ and $\sim 0.02 \mathrm{mag}$ in $(\mathrm{B}-\mathrm{V}),(\mathrm{V}-\mathrm{R})$ and $(\mathrm{V}-\mathrm{I})$ while $\sim 0.03 \mathrm{mag}$ in $(\mathrm{U}-\mathrm{B})$. The JHK data set have error $\sim 0.05 \mathrm{mag}$ in $\mathrm{J}$ mag.

\section{INTERSTELLAR EXTINCTION LAW}

\section{A. Determination of $\mathrm{R}_{\text {cluster }}$ value}

We have calculated a parameter ' $r$ ', to see the extent at which the clusters under study follow the normal interstellar extinction law. ' $r$ ' is the ratio of observed colour excess ratio and normal colour excess ratio as defined by [11], which can be expressed as 


\section{International Advanced Research Journal in Science, Engineering and Technology}

Vol. 6, Issue 5, May 2019

$$
\frac{[\mathrm{E}(\mathrm{V}-\lambda) / \mathrm{E}(\mathrm{B}-\mathrm{V})]_{\text {cluster }}}{[\mathrm{E}(\mathrm{V}-\lambda) / \mathrm{E}(\mathrm{B}-\mathrm{V})]_{\text {normal }}}
$$

\begin{tabular}{|c|c|c|c|c|c|c|c|}
\hline \multirow[t]{2}{*}{ Cluster } & \multirow[t]{2}{*}{$\mathrm{R}_{\text {cluster }}$} & \multicolumn{6}{|c|}{ ' $\mathrm{r}$ ' corresponding to $\lambda^{-1}\left(\mu \mathrm{m}^{-1}\right)$} \\
\hline & & $2.90(\mathrm{U})$ & $1.56(\mathrm{R})$ & $1.25(\mathrm{I})$ & $0.80(\mathrm{~J})$ & $0.61(\mathrm{H})$ & $0.45(\mathrm{~K})$ \\
\hline Berkeley 7 & $2.89 \pm 0.18$ & $1.00 \pm 0.02$ & $1.11 \pm 0.04$ & $0.94 \pm 0.04$ & $0.86 \pm 0.05$ & $0.96 \pm 0.05$ & $0.93 \pm 0.04$ \\
\hline NGC 6823 & $2.95 \pm 0.05$ & $1.01 \pm 0.02$ & $1.11 \pm 0.09$ & $1.11 \pm 0.12$ & $0.91 \pm 0.10$ & $0.94 \pm 0.07$ & $0.94 \pm 0.07$ \\
\hline Collinder 232 & $3.67 \pm 0.06$ & $1.09 \pm 0.03$ & $1.03 \pm 0.09$ & $1.09 \pm 0.10$ & $1.10 \pm 0.16$ & $1.15 \pm 0.20$ & $1.14 \pm 0.20$ \\
\hline NGC 6530 & $4.70 \pm 0.16$ & $1.08 \pm 0.09$ & $1.35 \pm 0.25$ & $1.25 \pm 0.12$ & $1.39 \pm 0.05$ & $1.37 \pm 0.17$ & $1.50 \pm 0.17$ \\
\hline Haffner 19 & $3.35 \pm 0.40$ & $1.00 \pm 0.05$ & $1.01 \pm 0.07$ & $1.13 \pm 0.08$ & $0.91 \pm 0.10$ & $1.12 \pm 0.06$ & $1.11 \pm 0.10$ \\
\hline NGC 7160 & $2.76 \pm 0.22$ & $1.16 \pm 0.11$ & $1.06 \pm 0.23$ & $1.08 \pm 0.18$ & $0.93 \pm 0.12$ & $0.81 \pm 0.13$ & $0.89 \pm 0.09$ \\
\hline NGC 6193 & $3.11 \pm 0.29$ & $1.08 \pm 0.10$ & $1.13 \pm 0.12$ & $1.27 \pm 0.14$ & $0.88 \pm 0.16$ & $1.03 \pm 0.17$ & $1.02 \pm 0.19$ \\
\hline Bochum 10 & $3.18 \pm 0.40$ & $1.38 \pm 0.17$ & $1.06 \pm 0.25$ & $1.12 \pm 0.44$ & $0.94 \pm 0.27$ & $0.91 \pm 0.23$ & $1.15 \pm 0.24$ \\
\hline Haffner 18 & $3.20 \pm 0.33$ & $1.02 \pm 0.02$ & $1.01 \pm 0.06$ & $1.04 \pm 0.07$ & $0.89 \pm 0.10$ & $1.07 \pm 0.05$ & $1.05 \pm 0.08$ \\
\hline NGC 2362 & $3.75 \pm 0.65$ & $1.30 \pm 0.13$ & $0.78 \pm 0.21$ & $0.92 \pm 0.22$ & $1.04 \pm 0.37$ & $1.03 \pm 0.30$ & $1.41 \pm 0.26$ \\
\hline Hogg 10 & $3.44 \pm 0.41$ & $1.15 \pm 0.03$ & $1.00 \pm 0.13$ & $1.11 \pm 0.07$ & $0.90 \pm 0.10$ & $0.87 \pm 0.14$ & $0.95 \pm 0.16$ \\
\hline NGC 2401 & $3.44 \pm 0.41$ & $1.06 \pm 0.08$ & $1.28 \pm 0.18$ & $1.22 \pm 0.14$ & $1.13 \pm 0.15$ & $1.11 \pm 0.10$ & $0.94 \pm 0.11$ \\
\hline IC 4996 & $2.61 \pm 0.38$ & $1.01 \pm 0.05$ & $0.55 \pm 0.10$ & $1.48 \pm 0.09$ & $0.73 \pm 0.13$ & $0.84 \pm 0.16$ & $0.94 \pm 0.16$ \\
\hline NGC 2384 & $3.06 \pm 0.24$ & $0.95 \pm 0.12$ & $0.55 \pm 0.46$ & $1.87 \pm 0.69$ & $1.01 \pm 0.25$ & $0.88 \pm 0.15$ & $0.99 \pm 0.11$ \\
\hline Czernik 43 & $2.32 \pm 0.31$ & $1.03 \pm 0.03$ & $0.90 \pm 0.16$ & $*$ & $0.67 \pm 0.10$ & $0.81 \pm 0.09$ & $0.79 \pm 0.09$ \\
\hline NGC 6871 & $2.82 \pm 0.07$ & $0.97 \pm 0.03$ & * & * & $0.87 \pm 0.11$ & $0.91 \pm 0.07$ & $0.89 \pm 0.07$ \\
\hline NGC 6618 & $2.92 \pm 0.30$ & $1.11 \pm 0.03$ & $*$ & $*$ & $0.83 \pm 0.09$ & $0.93 \pm 0.20$ & $1.01 \pm 0.29$ \\
\hline NGC 7380 & $3.12 \pm 0.21$ & $1.02 \pm 0.02$ & * & * & $0.91 \pm 0.07$ & $1.03 \pm 0.05$ & $1.00 \pm 0.06$ \\
\hline NGC 6231 & $3.12 \pm 0.15$ & $1.07 \pm 0.02$ & $*$ & $0.99 \pm 0.02$ & $0.93 \pm 0.07$ & $1.02 \pm 0.05$ & $0.98 \pm 0.05$ \\
\hline Collinder 69 & $3.57 \pm 0.82$ & $1.04 \pm 0.05$ & * & * & $1.06 \pm 0.24$ & $0.89 \pm 0.13$ & $1.38 \pm 0.12$ \\
\hline
\end{tabular}

Table I The Value of Parameter ' $R$ ' Corresponding to $\Lambda^{-1}$ for Twenty Open Star Clusters in Wave Band Urijhk.

The values of observed colour-excess ratios for all the clusters are taken from [10]. The calculated values of ' $r$ ' for all the clusters are listed in Table 1 along with the value of $\lambda^{-1}$ in the optical and near-IR waveband. The value of ' $r$ ' is plotted against $\lambda^{-1}$ in Fig. 1. The horizontal line in this figure denotes the value $r=1$ for which clusters follow the normal extinction law. [1] studied interstellar extinction law for different wavelength range and found that for the wavelength range $0.32>\lambda>0.12 \mu \mathrm{m}$, the total to selective extinction ratio $A_{\lambda} / A_{V}$ can be represented as

$$
\frac{A_{\lambda}}{A_{V}}=a_{\lambda}+b_{\lambda} / R
$$

where $a_{\lambda}$ and $b_{\lambda}$ are wavelength dependent constants. The value of these constants can be calculated by using the relations given in [1]. In this study we have used the relation given by [1] for the wavelength range $0.3 \mu \mathrm{m}^{-1} \leq \mathrm{x} \leq$ $1.1 \mu \mathrm{m}^{-1}$ and calculated the value of $\mathrm{R}_{\text {cluster }}$ using the above formula in the following manner:

$$
\frac{E(\lambda-V)}{E(B-V)}=R_{\text {cluster }}\left(a_{\lambda}+1\right)+b_{\lambda}
$$




\section{International Advanced Research Journal in Science, Engineering and Technology}

Vol. 6, Issue 5, May 2019

where $R_{\text {cluster }}$ is the total to selective extinction towards the direction of cluster. The calculated values of $R_{\text {cluster }}$ for every cluster are listed in Table 1 . The value of total to selective extinction for the general interstellar medium in the wavelength range $0.3 \leq \mathrm{x} \leq 1.1 \mu \mathrm{m}^{-1}$ is 3.1 ([1], [11]). Our analysis shows that fifteen clusters in the sample have value of $\mathrm{R}_{\text {cluster }} \sim 3.1$ within error so they have same grain properties as general interstellar medium. The remaining five clusters show anomalous values in the sense that two clusters Collinder 232 and NGC 6530 have values larger than 3.1 so the dust grains which are responsible for the extinction in the direction of these clusters are larger in size than the general interstellar medium. There is also a possibility of dense interstellar medium or circumstellar dust around these clusters ([15]). Three clusters NGC 7160, NGC 6871 and Czernik 43 have $\mathrm{R}_{\text {cluster }}$ values less than the normal value, which indicates that grains must have smaller size ([15]).
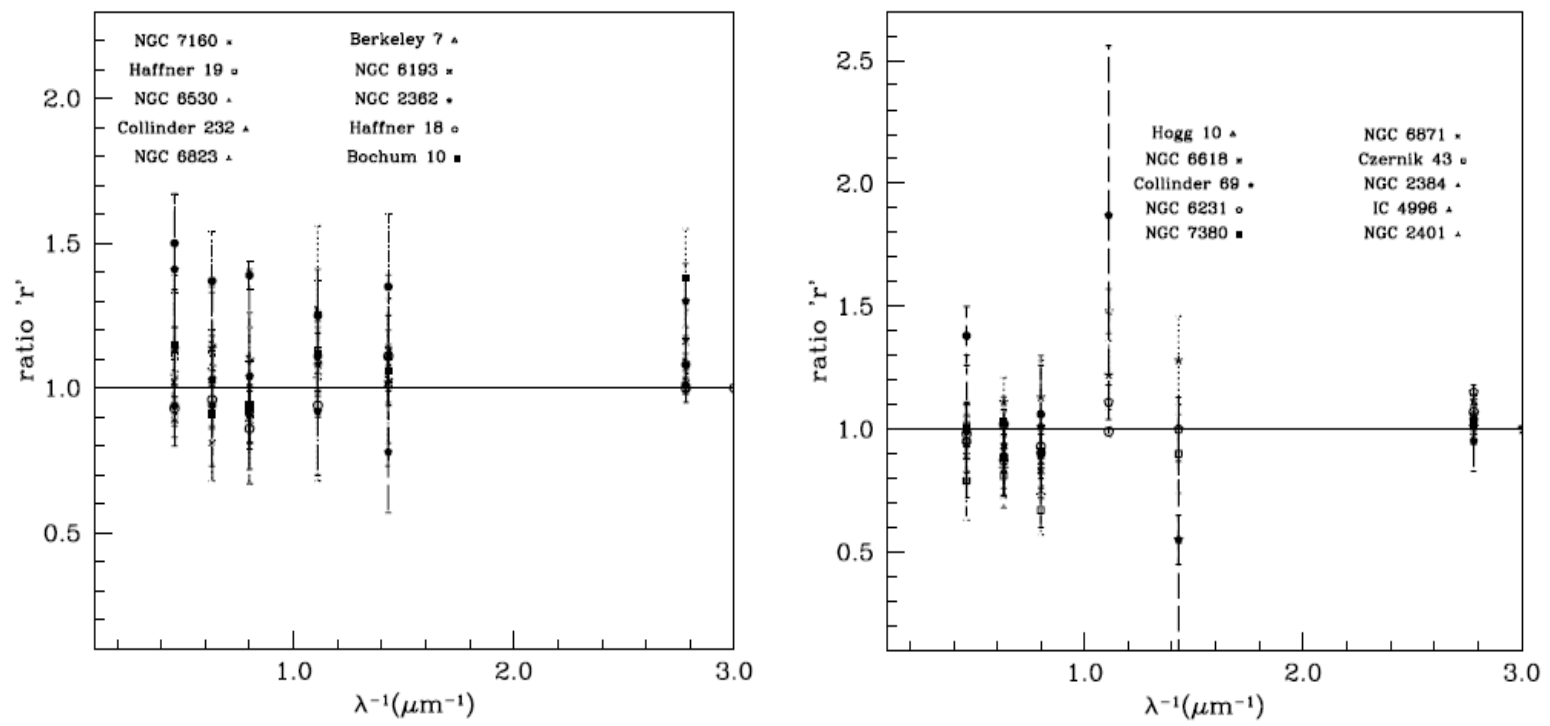

Fig. 1 The value of ' $r$ ' is plotted against $\lambda^{-1}$ for each cluster under study. The solid line represents value ' $r$ ' $=1$.

The standard deviations from mean value are denoted by the error bars.

B. $A_{\lambda} / A_{V}$ curves

Generally the extinction law is studied as the ratio of two colour excesses such as $E(V-\lambda) / E(B-V)$. But according to [1], on normalizing the colour excess ratio, the true nature of extinction may remain hidden. The true nature of extinction law can be analyzed by the quantity $R\left(=A_{\lambda} / A_{V}\right)$. As stated by [9] the quantity $A_{\lambda} / A_{V}$ can be calculated by the relation given below.

$$
\frac{A_{\lambda}}{A_{V}}=\left[\frac{E(\lambda-V)}{E(B-V) R_{\text {cluster }}}\right]+1
$$

We have plotted the values of $A_{\lambda} / A_{V}$ against $\lambda^{-1}$ in Fig. 2 for all the clusters under study. In the present analysis, fifteen clusters have $R_{\text {cluster }} \sim 3.1$. This indicates that these clusters have normal behavior according to [1]. In the left panel of Fig. 2, we have plotted $A_{\lambda} / A_{V}$ against $\lambda^{-1}$ for the clusters Haffner 19, NGC 6193, Bochum 10, Hogg 10, NGC 2401, IC 4996, NGC 2384, Berkeley 7, NGC 6823, Haffner 18, NGC 2362, NGC 6231, NGC 6618, NGC 7380 and Collinder 69 and in the right panel of this figure, same graph has been plotted for the clusters NGC 7160, Collinder 232, NGC 6530, Czernik 43 and NGC 6871 for which $R_{\text {cluster }}$ deviates from $\mathrm{R}=3.1$.

We have also plotted the normal value of $R_{\text {cluster }}=3.1$ ([1]) with solid line. This figure shows that the value of $A_{\lambda} / A_{V}$ for fifteen clusters are very similar to normal value within the error for $\lambda^{-1} \geq 1.43$. The value of $A_{\lambda} / A_{V}$ lies on the solid line. This means that all these fifteen clusters follow normal extinction law for $\lambda^{-1} \geq 1.43$. For the clusters NGC 7160, Collinder 232, NGC 6530, Czernik 43 and NGC 6871 the values of $A_{\lambda} / A_{V}$ show deviation from normal extinction law. Therefore for these clusters we have calculated the coefficients $a_{\lambda}$ and $b_{\lambda}$ by considering the relations given by [1] and using the corresponding $R_{\text {cluster }}$ values listed in Table 1 . In right panel of Fig. 2 we have plotted $A_{\lambda} / A_{V}$ against $\lambda^{-1}$ using the coefficients $a_{\lambda}$ and $b_{\lambda}$ with the different types of lines. In this figure we can see that except Czernik 43 all four clusters within error follow the corresponding $R_{\text {cluster }}$ value in each wavelength. In the optical range the value of $A_{\lambda} / A_{V}$ follow the corresponding $R_{\text {cluster }}$ value but in near IR wavelength the values are less than the values of $R_{\text {cluster }}$ as calculated by us using the formula derived by [1]. 


\section{International Advanced Research Journal in Science, Engineering and Technology}

Vol. 6, Issue 5, May 2019

Table II The Value of $A_{\lambda} / A_{V}$ Corresponding to $\mathrm{R}=3.1$ for the Clusters Having Normal $\mathrm{R}_{\text {cluster }}$ Value in each wave band. The value of $\lambda^{-1}$ are also Listed.

\begin{tabular}{|c|c|c|c|c|c|c|c|c|c|}
\hline \multirow{2}{*}{$\begin{array}{c}\lambda^{-1} \\
\left(\mu \mathrm{m}^{-1}\right)\end{array}$} & \multicolumn{8}{|c|}{$\mathrm{A}_{\lambda} / \mathrm{A}_{\mathrm{V}}$} & \multirow[b]{2}{*}{$\begin{array}{l}\text { Berkeley } \\
7\end{array}$} \\
\hline & $\begin{array}{l}\text { Normal } \\
\text { value }\end{array}$ & Haffner 19 & $\begin{array}{l}\text { NGC } \\
6193\end{array}$ & $\begin{array}{l}\text { Bochum } \\
10\end{array}$ & Hogg 10 & $\begin{array}{l}\text { NGC } \\
2401\end{array}$ & IC 4996 & $\begin{array}{l}\text { NGC } \\
2384\end{array}$ & \\
\hline $2.78(\mathrm{U})$ & 1.569 & $\begin{array}{l}1.51 \pm \\
0.19\end{array}$ & $\begin{array}{l}1.59 \pm \\
0.21\end{array}$ & $\begin{array}{l}1.74 \pm \\
0.31\end{array}$ & $\begin{array}{l}1.57 \pm \\
0.19\end{array}$ & $\begin{array}{l}1.53 \pm \\
0.21\end{array}$ & $\begin{array}{l}1.66 \pm \\
0.26\end{array}$ & $\begin{array}{l}1.53 \pm \\
0.22\end{array}$ & $\begin{array}{l}1.59 \pm \\
0.10\end{array}$ \\
\hline 2.37 (B) & 1.337 & $\begin{array}{l}1.29 \pm \\
0.40\end{array}$ & $\begin{array}{l}1.32 \pm \\
0.29\end{array}$ & $\begin{array}{l}1.79 \pm \\
0.40\end{array}$ & $\begin{array}{l}1.29 \pm \\
0.41\end{array}$ & $\begin{array}{l}1.29 \pm \\
0.41\end{array}$ & $\begin{array}{l}1.38 \pm \\
0.38\end{array}$ & $\begin{array}{l}1.32 \pm \\
0.24\end{array}$ & $\begin{array}{l}1.34 \pm \\
0.18\end{array}$ \\
\hline $1.82(\mathrm{~V})$ & 1 & 1 & 1 & 1 & 1 & 1 & 1 & 1 & 1 \\
\hline $1.42(\mathrm{R})$ & 0.751 & $\begin{array}{l}0.07 \pm \\
0.01\end{array}$ & $\begin{array}{l}0.78 \pm \\
0.10\end{array}$ & $\begin{array}{l}0.79 \pm \\
0.21\end{array}$ & $\begin{array}{l}0.82 \pm \\
0.14\end{array}$ & $\begin{array}{l}0.77 \pm \\
0.14\end{array}$ & $\begin{array}{l}0.87 \pm \\
0.20\end{array}$ & $\begin{array}{l}0.89 \pm \\
0.76\end{array}$ & $\begin{array}{l}0.76 \pm \\
0.05\end{array}$ \\
\hline $1.11(\mathrm{I})$ & 0.479 & $\begin{array}{l}0.13 \pm \\
0.01\end{array}$ & $\begin{array}{l}0.48 \pm \\
0.07\end{array}$ & $\begin{array}{l}0.56 \pm \\
0.23\end{array}$ & $\begin{array}{l}0.59 \pm \\
0.08\end{array}$ & $\begin{array}{l}0.55 \pm \\
0.09\end{array}$ & $\begin{array}{l}0.28 \pm \\
0.04\end{array}$ & $\begin{array}{l}0.23 \pm \\
0.08\end{array}$ & $\begin{array}{l}0.59 \pm \\
0.04\end{array}$ \\
\hline $0.80(\mathrm{~J})$ & 0.282 & $\begin{array}{l}0.37 \pm \\
0.06\end{array}$ & $\begin{array}{l}0.34 \pm \\
0.07\end{array}$ & $\begin{array}{l}0.31 \pm \\
0.10\end{array}$ & $\begin{array}{l}0.59 \pm \\
0.08\end{array}$ & $\begin{array}{l}0.23 \pm \\
0.04\end{array}$ & $\begin{array}{l}0.35 \pm \\
0.08\end{array}$ & $\begin{array}{l}0.23 \pm \\
0.06\end{array}$ & $\begin{array}{l}0.31 \pm \\
0.02\end{array}$ \\
\hline $0.63(\mathrm{H})$ & 0.190 & $\begin{array}{l}0.57 \pm \\
0.07\end{array}$ & $\begin{array}{l}0.13 \pm \\
0.26\end{array}$ & $\begin{array}{l}0.25 \pm \\
0.07\end{array}$ & $\begin{array}{l}0.34 \pm \\
0.06\end{array}$ & $\begin{array}{l}0.16 \pm \\
0.02\end{array}$ & $\begin{array}{l}0.16 \pm \\
0.04\end{array}$ & $\begin{array}{l}0.25 \pm \\
0.47\end{array}$ & $\begin{array}{l}0.14 \pm \\
0.01\end{array}$ \\
\hline $0.46(\mathrm{~K})$ & 0.114 & $\begin{array}{l}0.81 \pm \\
0.11\end{array}$ & $\begin{array}{l}0.08 \pm \\
0.01\end{array}$ & $\begin{array}{l}0.00 \pm \\
0.007\end{array}$ & $\begin{array}{l}0.22 \pm \\
0.04\end{array}$ & $\begin{array}{l}0.23 \pm \\
0.04\end{array}$ & $\begin{array}{l}0.00 \pm \\
0.001\end{array}$ & $\begin{array}{l}0.09 \pm \\
0.01\end{array}$ & $\begin{array}{l}0.09 \pm \\
0.007\end{array}$ \\
\hline
\end{tabular}

Table III The Value OF $A_{\lambda} / A_{V}$ Corresponding to $R=3.1$ for the Clusters Having Normal $R_{\text {cluster }}$ Value in each wave band. The value of $\lambda^{-1}$ are also Listed.

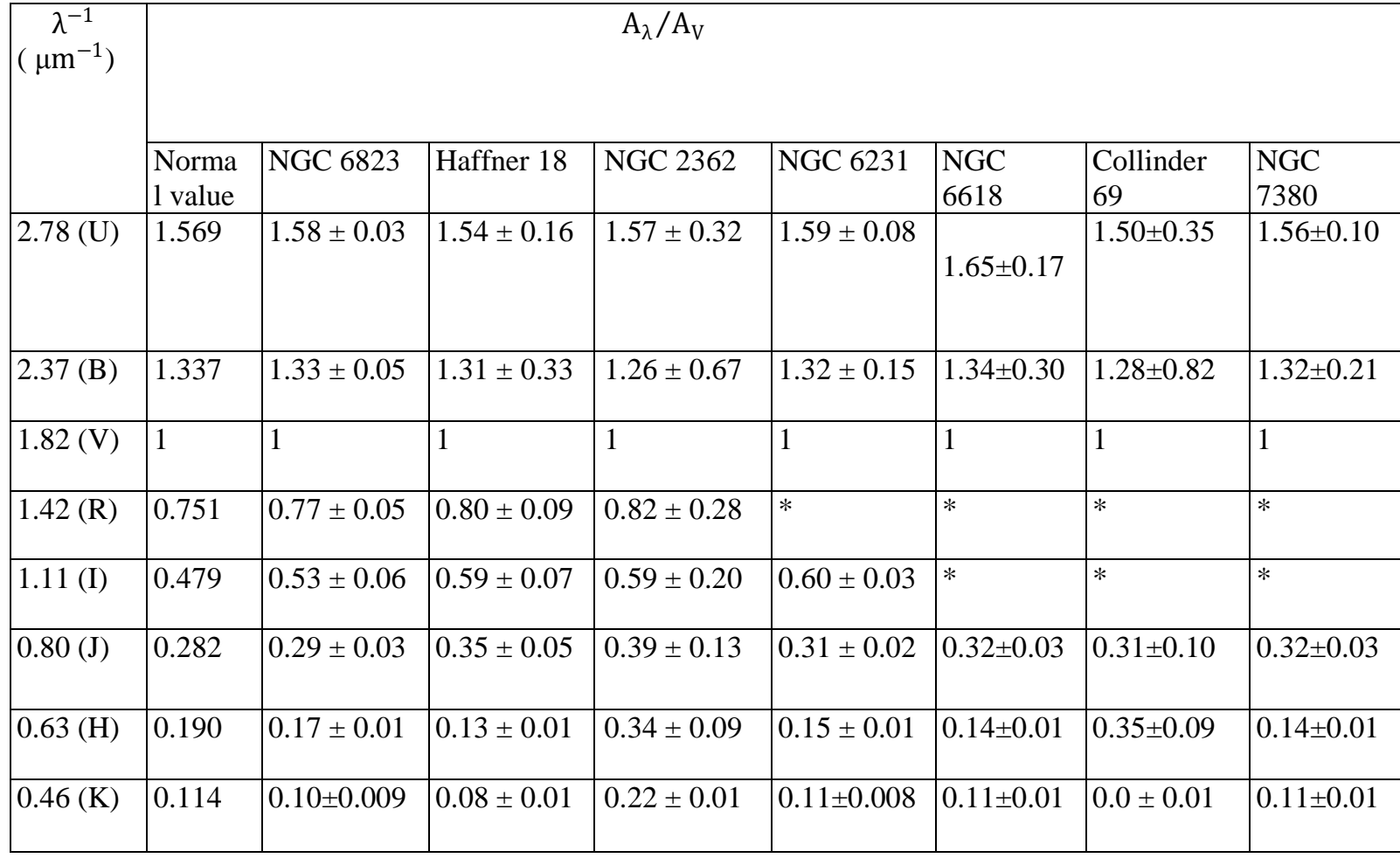




\section{International Advanced Research Journal in Science, Engineering and Technology}

Vol. 6, Issue 5, May 2019

Table IV Value of $A_{\lambda} / A_{V}$ Corresponding to $R_{\text {cluster }}$ for Five Clusters Having Anomalous $R_{\text {cluster }}$ Value.

\begin{tabular}{|c|c|c|c|c|c|c|c|c|c|c|}
\hline \multirow{2}{*}{$\begin{array}{c}\lambda^{-1} \\
\left(\mu \mathrm{m}^{-1}\right)\end{array}$} & \multicolumn{2}{|c|}{ NGC 7160} & \multicolumn{2}{|c|}{ Collinder 232} & \multicolumn{2}{|c|}{ NGC 6530} & \multicolumn{2}{|c|}{ Czernik 43} & \multicolumn{2}{|c|}{ NGC 6871} \\
\hline & $\mathrm{R}_{\text {cluster }}$ & $\mathrm{A}_{\lambda} / \mathrm{A}_{\mathrm{V}}$ & $\mathrm{R}_{\text {cluster }}$ & $\mathrm{A}_{\lambda} / \mathrm{A}_{\mathrm{V}}$ & $\mathrm{R}_{\text {cluster }}$ & $A_{\lambda} / A_{V}$ & $\mathrm{R}_{\text {cluster }}$ & $\mathrm{A}_{\lambda} / \mathrm{A}_{\mathrm{V}}$ & $\mathrm{R}_{\text {cluster }}$ & $\mathrm{A}_{\lambda} / \mathrm{A}_{\mathrm{V}}$ \\
\hline $2.78(\mathrm{U})$ & 1.644 & $\begin{array}{l}1.723 \pm \\
0.21\end{array}$ & 1.473 & $\begin{array}{l}1.512 \pm \\
0.05\end{array}$ & 1.359 & $\begin{array}{l}1.397 \pm \\
0.13\end{array}$ & 1.775 & $\begin{array}{l}1.764 \pm \\
0.24\end{array}$ & 1.629 & $\begin{array}{l}1.594 \pm \\
0.06\end{array}$ \\
\hline $2.27(\mathrm{~B})$ & 1.378 & $\begin{array}{l}1.362 \pm \\
0.22\end{array}$ & 1.284 & $\begin{array}{l}1.272 \pm \\
0.06\end{array}$ & 1.221 & $\begin{array}{l}1.212 \pm \\
0.16\end{array}$ & 1.450 & $\begin{array}{l}1.431 \pm \\
0.31\end{array}$ & 1.370 & $\begin{array}{l}1.354 \pm \\
0.07\end{array}$ \\
\hline $1.82(\mathrm{~V})$ & 1 & 1 & 1 & 1 & 1 & 1 & 1 & 1 & 1 & 1 \\
\hline $1.43(\mathrm{R})$ & 0.735 & \begin{tabular}{|l|}
$0.768 \pm$ \\
0.24
\end{tabular} & 0.770 & $\begin{array}{l}0.831 \pm \\
0.06\end{array}$ & 0.792 & $\begin{array}{l}0.827 \pm \\
0.15 \\
\end{array}$ & 0.713 & $\begin{array}{l}0.768 \pm \\
0.17\end{array}$ & 0.740 & $*$ \\
\hline $1.11(\mathrm{I})$ & 0.453 & \begin{tabular}{|l|}
$0.511 \pm$ \\
0.09 \\
\end{tabular} & 0.51 & $\begin{array}{l}0.626 \pm \\
0.06\end{array}$ & 0.547 & \begin{tabular}{|l}
$0.665 \pm$ \\
0.07 \\
\end{tabular} & 0.411 & $*$ & 0.458 & $*$ \\
\hline $0.80(\mathrm{~J})$ & 0.267 & $\begin{array}{l}0.225 \pm \\
0.03\end{array}$ & 0.300 & $\begin{array}{l}0.305 \pm \\
0.04\end{array}$ & 0.322 & $\begin{array}{l}0.317 \pm \\
0.01\end{array}$ & 0.242 & $\begin{array}{l}0.334 \pm \\
0.06\end{array}$ & 0.270 & $\begin{array}{l}0.285 \pm \\
0.03\end{array}$ \\
\hline $0.63(\mathrm{H})$ & 0.179 & \begin{tabular}{|l}
$0.244 \pm$ \\
0.04
\end{tabular} & 0.201 & $\begin{array}{l}0.188 \pm \\
0.03\end{array}$ & 0.216 & $\begin{array}{l}0.244 \pm \\
0.03\end{array}$ & 0.162 & $\begin{array}{l}0.098 \pm \\
0.01\end{array}$ & 0.181 & $\begin{array}{l}0.161 \pm \\
0.01\end{array}$ \\
\hline $0.46(\mathrm{~K})$ & 0.107 & $\begin{array}{l}0.095 \pm \\
0.01 \\
\end{array}$ & 0.121 & $\begin{array}{l}0.133 \pm \\
0.02\end{array}$ & 0.129 & $\begin{array}{l}0.108 \pm \\
0.01\end{array}$ & 0.097 & $\begin{array}{l}0.046 \pm \\
0.008\end{array}$ & 0.108 & $\begin{array}{l}0.115 \pm \\
0.009\end{array}$ \\
\hline
\end{tabular}

\section{CONCLUSION}

In the present analysis we studied the interstellar extinction in the direction of twenty young open clusters. We calculated the total to selective extinction ratio $\mathrm{R}_{\text {cluster }}$ for these clusters. From this analysis we found that-

1. The calculated $R_{\text {cluster }}$ value indicates that fifteen clusters have normal values; it means the grain size of the dust in the direction of these clusters is same as the general interstellar medium. Two clusters Collinder 232 and NGC 6530 have $\mathrm{R}_{\text {cluster }}$ values greater than 3.1, it means interstellar dust responsible of extinction in the direction of these clusters have larger size than that of normal interstellar dust. Three clusters NGC 7160, NGC 6871 and Czernik 43 have value less than 3.1; it means dust grains in the direction of these clusters have smaller size than general interstellar medium.

2. Present analysis shows that clusters which have normal $R_{\text {cluster }}$ value show normal extinction law for $\lambda^{-1} \geq 1.43$ and below this value of $\lambda^{-1}$ they show abnormal behavior from the normal extinction law. Our analysis also shows that except Czernik 43 which shows anomaly in near-IR region all clusters fall on the line derived by using $R_{\text {cluster }}$ value.

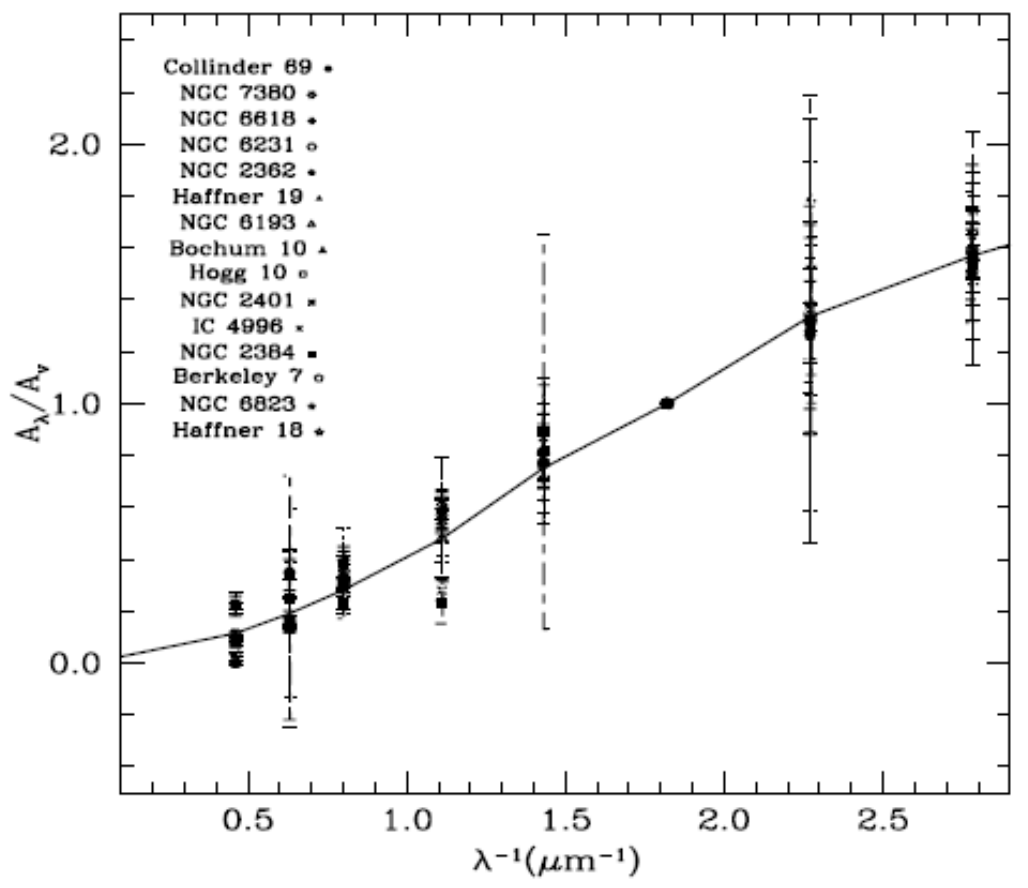




\section{International Advanced Research Journal in Science, Engineering and Technology}

Vol. 6, Issue 5, May 2019

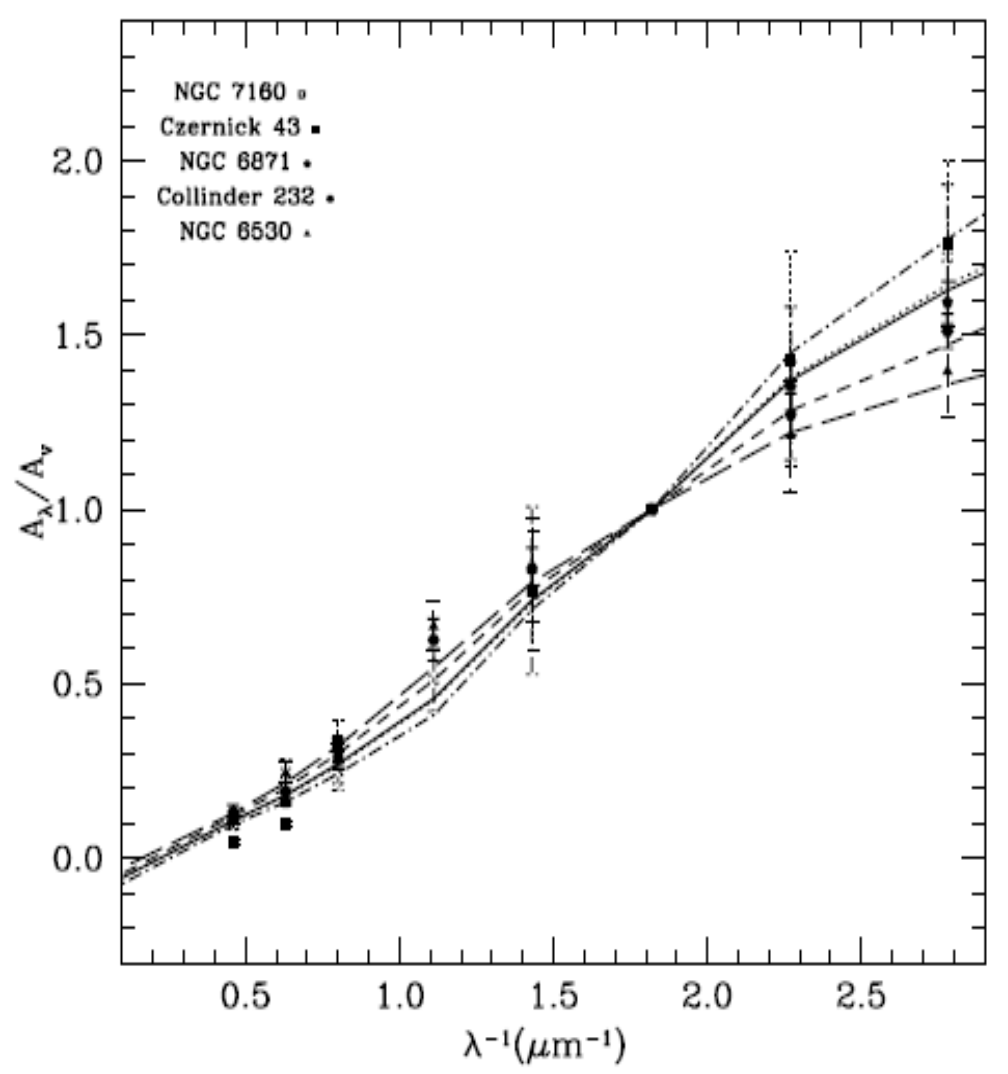

Fig. 2 The $A_{\lambda} / A_{V}$ normalized extinction curve for all twenty clusters with the solid line representing the value of $R_{\text {cluster }}$. The bars represent standard deviation from the mean value.

\section{ACKNOWLEDGEMENT}

This research has made use of the WEBDA database, operated at the Department of Theoretical Physics and Astrophysics of the Masaryk University. We have also used data from Two Micron All Sky Survey, which is a joint project of the University of Massachusetts and the Infrared Processing and Analysis Center/California Institute of Technology, funded by the National Aeronautics and Space Administration and the National Science Foundation.

\section{REFERENCES}

[1]. Cardelli, J.A, Clayton, G.C., Mathis J.S, 1989. The relationship between infrared, optical and ultraviolet extinction. ApJ, 345, 245-256.

[2]. Fitzpatrick, E. L., 2004. Interstellar Extinction in the Milky Way Galaxy. ASP Conference Series, Vol.309.

[3]. Johnson, H. L. \& Morgan, W. W., 1953. Fundamental stellar photometry for standards of spectral type on the Revised System of the Yerkes Spectral Atlas.. ApJ, 117, 313-352.

[4]. Joshi, Y. C., 2005. Interstellar extinction towards open clusters and Galactic structure. MNRAS, 362, 1259-1266.

[5]. Hayashi, C., 1970. Collapse and Contraction to the Main Sequence, Introductory Report. Mem. Soc. R. Sci. Liege, $19,127$.

[6]. Kiszkurno, E., Kolos, R., Krelowski, J. \& Strobel, A., 1984. The distribution of the extinction in the galactic disc. A \& A, 135, 337-340

[7]. Krelowski, J. \& Strobel, A., 1983. A comparison of UV extinction in SCO OB2 and Per OB1 associations. A\&A, 127, 271-276.

[8]. Larson, R. B., 1973. Processes in Collapsing Interstellar Clouds. ARA \& A, 11, 219-238.

[9]. Martin, P. G. \& Whittet, D. C. B., 1990. Interstellar extinction and polarization in the infrared. ApJ, 357, 113-124.

[10]. McNamara, B. J., 1976. Pre-main-sequence masses and the age spread in the Orion cluster. AJ, 81, 845-854.

[11]. Pandey, A. K., Upadhyay K., Nakada, Y. \& K. Ogura, K., 2003. Interstellar extinction in the open clusters towards galactic longitude around $130^{\circ}$. A \& A, 397, 191-200.

[12]. Rangwal, G., Yadav, R. K., Durgapal, A. K. \& Bisht, D., 2017. Interstellar Extinction in 20 Open Star Clusters. PASA, 43, e068-e080.

[13]. Tapia, M., Costero, R., Echevarria, R. M., 1991. Near-infrared and Stromgren photometry of the open clusters NGC 663, NGC 1502 and NGC 1893. MNRAS, 253, 649-661.

[14]. Trumpler, R. J., 1930. Preliminary results on the distances, dimensions and space distribution of open star clusters. Lich observatory Bulletin, 14, 154-188.

[15]. Turner, D. G., 1994. The interstellar extinction law. RMxAA, 29, 163-174.

[16]. Warner, J. W., Strom, S. E., \& Strom, K. M., 1977. Circumstellar shells in NGC 2264: a reevaluation. ApJ, 213, $427-437$.

[17]. Yadav, R. K. S. \& Sagar, R., 2001. Non-uniform extinction in young open star clusters MNRAS, 328, $370-380$. 